\title{
Quality of life measurement in women with cervical cancer: implications for Chinese cervical cancer survivors
}

\author{
Ying Chun Zeng, Shirley SY Ching, Alice Y Loke*
}

\begin{abstract}
Background: Women with cervical cancer now have relatively good 5-year survival rates. Better survival rates have driven the paradigm in cancer care from a medical illness model to a wellness model, which is concerned with the quality of women's lives as well as the length of survival. Thus, the assessment of quality of life among cervical cancer survivors is increasingly paramount for healthcare professionals. The purposes of this review were to describe existing validated quality of life instruments used in cervical cancer survivors, and to reveal the implications of quality of life measurement for Chinese cervical cancer survivors.

Methods: A literature search of five electronic databases was conducted using the terms cervical/cervix cancer, quality of life, survivors, survivorship, measurement, and instruments. Articles published in either English or Chinese from January 2000 to June 2009 were searched. Only those adopting an established quality of life instrument for use in cervical cancer survivors were included.

Results: A total of 11 validated multidimensional quality of life instruments were identified from 41 articles. These instruments could be classified into four categories: generic, cancer-specific, cancer site-specific and cancer survivor-specific instruments. With internal consistency varying from 0.68-0.99, the test-retest reliability ranged from 0.60-0.95 based on the test of the Pearson coefficient. One or more types of validity supported the construct validity. Although all these instruments met the minimum requirements of reliability and validity, the original versions of these instruments were mainly in English.

Conclusion: Selection of an instrument should consider the purpose of investigation, take its psychometric properties into account, and consider the instrument's origin and comprehensiveness. As quality of life can be affected by culture, studies assessing the quality of life of cervical cancer survivors in China or other non-English speaking countries should choose or develop instruments relevant to their own cultural context. There is a need to develop a comprehensive quality of life instrument for Chinese cervical cancer survivors across the whole survivorship, including immediately after diagnosis and for short- (less than 5 years) and long-term (more than 5 years) survivorship.
\end{abstract}

\section{Introduction}

Cervical cancer is one of the most common types of cancer in developing countries. With nearly 500000 women developing cervical cancer per year, China's estimated 131500 new cases constitute $28.8 \%$ of the total new cases annually worldwide [1]. Due to widespread screening programs, the majority of cervical cancer

\footnotetext{
* Correspondence: hsaloke@polyu.edu.hk
School of Nursing, The Hong Kong Polytechnic University, Hong Kong, PR

* Correspondence: hsaloke@polyu.edu.hk
School of Nursing, The Hong Kong Polytechnic University, Hong Kong, PR China
}

(c) 2010 Zeng et al; licensee BioMed Central Ltd. This is an Open Access article distributed under the terms of the Creative Commons Attribution License (http://creativecommons.org/licenses/by/2.0), which permits unrestricted use, distribution, and reproduction in any medium, provided the original work is properly cited.

cases are being diagnosed in the earlier stages. Along with new and advanced medical treatment, women with cervical cancer have relatively good 5-year survival rates. The overall 5-year survival rate of all stages of cervical cancer among Chinese women has been estimated to be 70.93\% [2].

Better survival rates have driven the paradigm in the life-altering burden of cancer care from a medical illness model to a wellness model concerned with the quality of women's lives as well as the length of survival [3]. The current reality of cancer therapies has also led us to 
recognize the significance of improving the quality of cancer survivors' lives [4]. Quality of life (QOL) is one of the health outcomes that enable healthcare providers to better address the ongoing concerns of cancer survivors.

Due to cultural differences, Chinese cancer survivors may have a different interpretation of QOL. The concept of QOL is defined by Western cancer survivors as being healthy and independent, reclaiming life, psychological well-being or social relationships [5]. Chinese cancer survivors view "normal living", a good working life, happiness, material resources and support from their families as essential indicators of QOL $[6,7]$.

As QOL in cancer survivors varies by treatment, time since diagnosis and cancer sites [8], there is a need to review QOL measurement issues with a focus on specific cancer sites. While Vistad et al. [9] reviewed studies about the impact of cervical cancer on women's QOL, their review revealed little about QOL measurement for this target population. Although Pearce et al. [10] and Zebrack \& Cella [11] conducted methodological reviews of QOL measurement in various types of cancer survivors, there is a lack of review articles focusing on QOL measurement in cervical cancer survivors.

\section{Aims}

The purpose of this review was to describe existing validated multidimensional QOL instruments used in cervical cancer survivors, and to reveal implications of QOL measurement for Chinese cervical cancer survivors.

\section{Framework of quality of life}

Quality of life is dynamic and changes over time [12]. Traditional models of QOL are a multidimensional construct of health including physical, psychological, social and spiritual well-being [13]. It has been argued that this traditional framework predominantly focuses on the individual-centered paradigm, and ignores contextual factors that influence QOL [14]. The contextual QOL model proposed by Ashing-Giwa [14] includes both the individual and systemic paradigms, and was adopted as the framework for this review.

Within each level of paradigm, there are four major domains and a variety of components. The individual level consists of (1) General Health domain including components of health status and co-morbidity; (2) Medical Factors domain including components of age at diagnosis and cancer characteristics; (3) Health Efficacy domain including components of health practices, utilization, perceived health efficacy and medical adherence; and (4) Psychological Well-being domain including components of emotional distress, cognitive function, and positive psychological feelings [14]. The systemic level consists of (1) Socio-ecological domain including components of socio-economic status, life burden, social support, and role/relationship changes; (2) Cultural domain including components of spirituality, acculturation, and interconnectedness; (3) Demographic domain including components of chronological age; and (4) Healthcare System domain including components of access to health care and satisfaction with the quality of health care [14].

\section{Methods}

\section{Searching strategies}

Articles published in English or in Chinese from January 2000 to June 2009 were searched for the review. Terms used for searching included cervical cancer, cervix cancer, survivors, survivorship, quality of life, measurement, assessment, and instruments, which were searched in five computerized databases: CINAHL, Medline, PsycInfo, Scopus, and the Chinese Journal Full-text Database (CJFD). In this review, the term 'cervical cancer survivor' was adapted to mean a person living with cervical cancer immediately after the initial diagnosis [15].

\section{The process of search and selections}

Initially, a total of 296 articles were identified from the literature search of the five databases using the above key words. Duplications of articles and those articles that did not meet the selection criteria were removed. Only 53 articles remained. Twelve of these had used self-designed instruments and did not report reliability and validity. As a result, a total of 41 articles were included. The flowchart of search and selection process was outlined in figure 1.

\section{Inclusion and exclusion criteria}

A checklist was used to select the literature. For inclusion, all of the following criteria had to be fulfilled by the articles: (1) QOL was one of primary outcome measures; (2) women with a diagnosis of cervical cancer constituted the study population; (3) papers were published either in English or in Chinese between January 2000 and June 2009 (at time of search). In terms of exclusion criteria, all qualitative studies, commentaries, editorials, literature reviews, and conference proceedings were excluded from this review. As the concept of QOL is multidimensional (including the physical, psychological, social and spiritual well-being dimensions) [13], studies focusing on a single domain of QOL only were excluded.

\section{Common types of reliability and validity in QOL measurement}

The basic characteristics of a good QOL instrument should demonstrate evidence of adequate reliability and validity [10]. The most common types of reliability 
Search terms used in computerized databases either singly or in combination Cervical/cervix cancer, survivors, quality of life, measur ${ }^{*}$, assessment, instruments

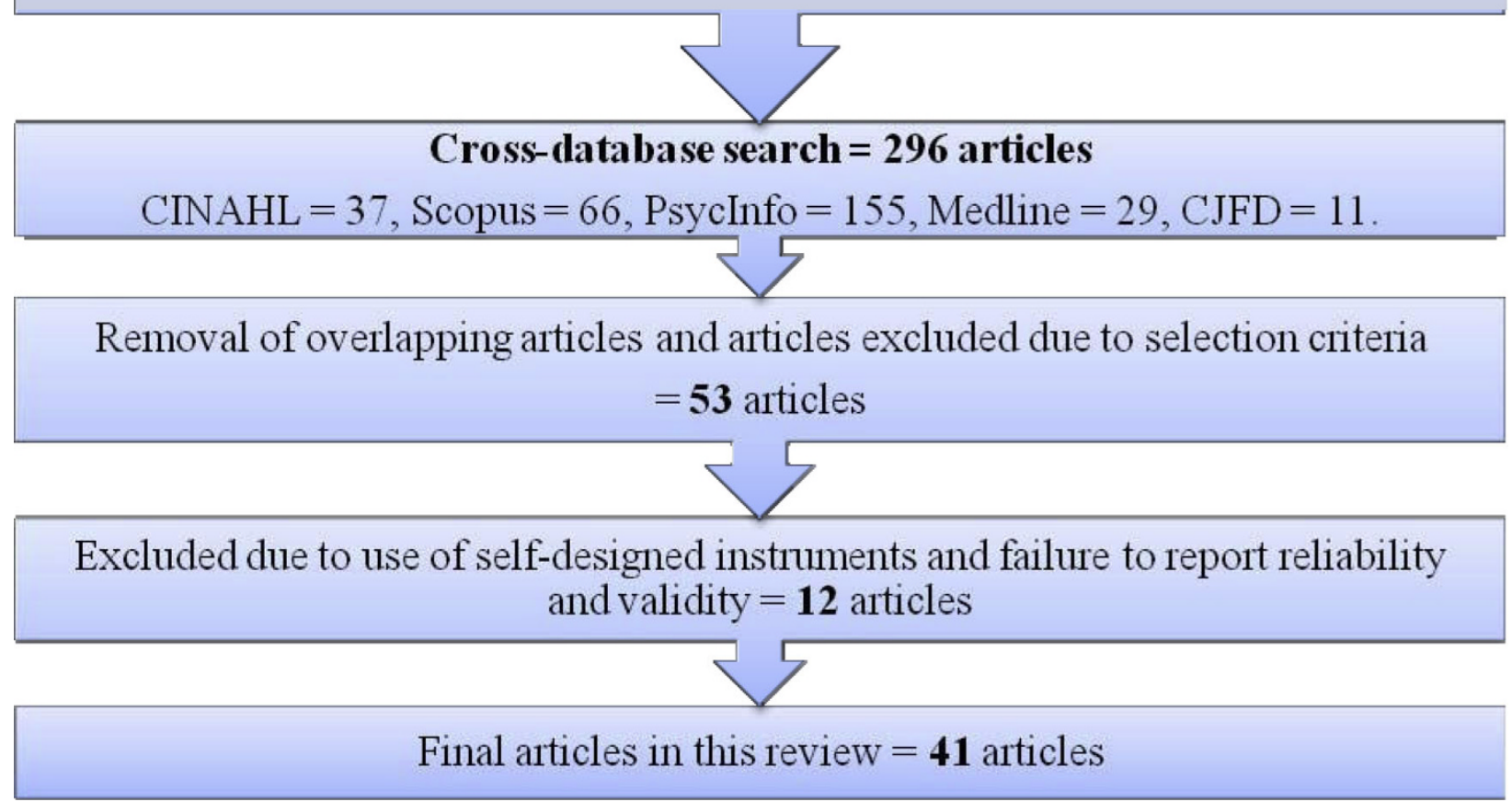

Figure 1 Flowchart of search and selection process.

reported for QOL questionnaires are internal consistency (assessing the homogeneity of the scale) and testretest reliability (assessing the stability of the scale) [16]; common types of validity reported by researchers include content validity (to what degree all items in a QOL instrument quantitatively represent the actual content area of the study) and construct validity (how well items reflect the latent variable in question), which can be assessed by convergent/divergent validation, knowngroup/contrasted-group validation and factor analysis approaches [17].

The minimum acceptable level of reliability and validity According to DeVellis [18], the acceptable level of internal consistency by Cronbach's alpha should be above 0.7. Fitzpatrick et al. [19] suggested that instruments examining test-retest reliability within 2-14 days and achieving a Pearson's correlation of over 0.7 were considered to be acceptable. If calculated by the Kappa coefficient or ICC (Intra-class Correlation Coefficient), an item total correlation of at least 0.2 is coded as acceptable [20]. In terms of construct validity, a convergent correlation score above 0.4 is coded as an acceptable standard [21]. By factor analysis, DeVellis [18] suggested that the eigenvalues of factors greater than 0.5 were considered to be acceptable. With known-group validity, the scale can differentiate among the groups [18].

\section{Results}

Among the 41 articles identified, 11 validated multidimensional instruments had been administered to assess QOL among cervical cancer survivors.

\section{Types of multidimensional QOL instruments}

After careful review of the characteristics and use of instruments in these studies, the instruments could be classified into four categories: generic instruments, cancer-specific instruments, cancer site-specific instruments, and survivor-specific instruments.

The generic questionnaires were designed to assess general aspects of QOL. This category included 4 instruments: the 36-item short form of the Medical Outcome Study questionnaire (SF-36) [22,23], the World Health Organization Quality of Life-Brief (WHOQOL-BREF) $[24,25]$, the Quality of Life Index (QLI) [26], and the European Quality of Life Scale-5 dimensions (EQ-5D) [27]. The cancer-specific instruments were designed to 
assess the QOL of cancer patients as a whole. This category contained 3 instruments: the Cancer Rehabilitation Evaluation System-Short Form (CARES-SF) [28], the European Organization for Research Treatment's Quality of Life Questionnaire (EORTC QLQ-C30) [29,30], and the Functional Assessment of Cancer Therapy-General (FACT-G) [31,32]. The cancer site-specific QOL instruments were developed to measure the QOL of cervical cancer patients. This category consisted of 3 scales: the EORTC Quality of Life Questionnaire-Cervix24items (QLQ-Cx24) [33], the Functional Assessment of Cancer Therapy-Cervix (FACT-Cx) [34,35], and the Quality of Life Instruments for Cancer Patients-Cervical Cancer (QLICP-CE) [36]. The survivor-specific category included the Cancer Survivors' Unmet Needs (CaSUN) scale [37], which was developed to assess QOL among long-term cancer survivors using a needs-based approach.

A brief description of each instrument, including categories, origin of countries and sample items, is shown in additional file 1 . While these instruments varied in length and emphasis, they shared the common perspective that QOL is a multidimensional concept including physical, psychological, social and spiritual well-being, and environmental conditions.

\section{The paradigms, domains, components and distribution of items}

There was a great variation in the domains and number of items in these 11 multidimensional QOL instruments. While these instruments were developed by different researchers and framed by different QOL models with combinations of related domains, it was considered beneficial to identify the common shared domains and components adopted to assess QOL among cervical cancer survivors. The item distribution of these 11 multidimensional instruments was tabulated according to AshingGiwa's contextual QOL model [14] (additional file 2).

Additional file 2 shows that, at the individual level, items in these QOL instruments mainly covered the domains of 'general health' and 'psychological health', with few covering 'medical factors' and 'health efficacy'. At the systemic level, these QOL instruments mainly included items to measure the socio-ecological domain, i.e. in the components of 'socio-economic status', 'social support', and 'role/relationship changes'. Very few items in these instruments covered the 'cultural domain' or the 'healthcare system'.

\section{The psychometric properties of multidimensional QOL instruments \\ Generic QOL instruments}

The SF-36 was developed by a medical outcomes health survey. Broadly, it consisted of 8 dimensions: physical functioning, role limitations due to physical health problems, bodily pain, general mental health covering psychological distress and well-being, role limitations due to emotional problems, social functioning, vitality, and general health perceptions [38]. The internal consistency for the overall scale was 0.95 [38]. The test-retest correlations were more than 0.8 in the physical function and general health perceptions domains [39]. Correlations of convergent validity between the SF-36 and the WHOQOL-BREF were: the physical component summary of SF-36 with the physical domains of WHOQOL-BREF was 0.48 ; and the mental component summary of SF-36 with the whole WHOQOL-BREF scale ranged from 0.6-0.75 [40].

The WHOQOL-BREF was a brief version of the QOL instrument developed from the WHOQOL-100. It comprised 26 items covering physical, psychological and social health, and environmental domains as well as overall QOL and health [41]. The internal consistency ranged from $0.75-0.86$ [40]. The test-retest reliability correlation ranged from $0.76-0.8$ in an interval of 2-4 weeks [42]. The content validity was assessed by assessing the item-domain correlations (0.53-0.78) and the inter-domain correlation (0.51-0.64) [42]. By convergent validation with SF-36, the mental health domain had a high correlation of 0.75 , and the lowest correlation in the physical functioning domain was 0.51 [40]. Factorial validity revealed 4 domains, and known-group validation differentiated the study population between sick and well individuals [43].

The QLI was designed to measure both the satisfaction and importance of various aspects of life, including the four domains of health and functioning, psychologi$\mathrm{cal} /$ spiritual, social and economic, and family [44]. This scale consisted of 66 items to rate for satisfaction and importance of QOL. The internal consistency alpha ranged from 0.73-0.99 [44]. The test-retest reliability was tested in a 2-week interval, and ranged from 0.68-0.79 [45]. Content validity was assessed by using the Content Validity Index, with an acceptable rating level [46]. By convergent validation with the Life Satisfaction Scale, the correlation ranged from 0.61-0.93; factor analysis derived 4 domains [45].

The EQ-5D consisted of 6 items covering 5 dimensions of health: mobility, self-care, usual activities, pain/ discomfort, and anxiety/depression, plus a global question to rate general health state [47]. The test-retest reliability was tested over a 1 -week interval and reported as 0.86 for group level coefficients averaged over health states [48]. The content validity was verified by the research panel. Using convergent validation with the Hospital Anxiety and Depression Scale, the correlation was reported respectively as 0.44 (Anxiety scale) and 0.51 (Depression scale) [49]. 


\section{Cancer-specific QOL instruments}

The CARES-SF contained 59 items, covering physical, psychological, medical interaction, marital, and sexual domains [50]. In Schag et al.'s validation study, the reliability of internal consistency had an estimated alpha ranging from 0.61-0.85, and the test-retest correlation was 0.92 with a 1 -month interval. The content validity of this scale was assessed by experts. Using convergent validation with the CARES, the correlation ranged from $0.67-0.85$. Factorial validity revealed 6 domains, and known-group validation was able to distinguish between normative and rehabilitation individuals [50].

The EORTC QLQ-C30 consisted of 30 items and included 5 functional domain scales, such as physical, role, emotional and social functions, along with disease-specific symptoms, a financial impact domain, and two items related to global health status and QOL [51]. The internal consistency with an estimated alpha ranged from 0.74-0.86 [51]. The test-retest correlation over a 4-day interval ranged from 0.82-0.91 [52]. By convergent validation with the CARES, the correlation was respectively reported as 0.46 (Social domain), 0.56 (Psychological domain), 0.69 (Pain symptoms), and 0.71 (Physical domain) [53].

The FACT-G included 27 items and covered 4 primary QOL domains: physical, emotional, social and functional well-being [54]. Cella et al.'s validation report shows an internal consistency alpha of 0.89 for the total instrument, and a test-retest correlation ranging from 0.82-0.92 over a 3- to 7-day interval. The convergent validation with the Functional Living Index-Cancer Scale was 0.79 . By using known-group validation, the FACT-G can significantly differentiate between patients at different stages of disease [54].

\section{Cancer site-specific QOL instruments}

The EORTC QLQ-Cx24 was developed to measure cervical cancer and its treatment-related issues. It covers the symptom experience, body image, and sexual/vaginal functioning subscales. The internal consistency of this scale ranged from 0.72-0.87 [55]. By convergent validation with the EORTC QLQ-C30, the correlation ranged from 0.4-0.48. The negative correlations of the body image subscale of QLQ-Cx24 with the emotional function and the global health/QOL of QLQ-C30 were minus 0.43 and 0.41 . Known-group validation could distinguish subgroups of patients based on their clinical status [55].

The FACT-Cx consisted of 42 items: 27 items from the FACT-G plus 15 additional items to measure specific cervical cancer concerns. It was translated into 27 languages for use among a group of cross-cultural cancer patients [16]. The internal consistency alpha for each domain ranged from 0.69-0.89 [56]. Known-group validation could distinguish subgroups of patients with different types of treatment [56].
The QLICP-CE consisted of 40 items covering 5 domains of QOL: physical function, psychological function, social function, common symptoms and sideeffects, and specific concerns of cervical cancer. Zhang et al. [36] reported that the internal consistency alpha for the overall scale was 0.68 and the test-retest reliability over a 3-day interval 0.95 . The content validity was verified by experts. The factor loading of all items that remained in the scale was at least 0.6 by factor analysis [36].

\section{QOL instruments for long-term cancer survivors}

The CaSUN was developed using a needs-based approach to assess QOL among cancer survivors. This instrument consisted of 35 items covering 5 domains: information and medical issues, QOL, emotional and relationship issues, life perspective, and positive change issues [37]. The internal consistency had an estimated alpha of 0.96. Based on a 3-week interval, the test-retest correlation by an estimation of the Kappa coefficient was 0.13 [37]. The content validity was verified by the research panel and feedback from respondents. By convergent validation with the Hospital Anxiety and Depression Scale, the correlation was respectively reported as 0.4 (Anxiety subscale), and 0.34 (Depression subscale) [37].

\section{Summaries of psychometric properties}

Additional file 3 also shows the psychometric properties of reliability and validity. The internal consistency of these 11 established multidimensional QOL instruments met the acceptable standards (0.68-0.99). In terms of the test-retest correlation, the average item-item correlation of CaSUN by Kappa coefficient was 0.13 , below the acceptable level of correlation. Although the test-retest reliability of QLICP-CE was 0.95 by Pearson's coefficient test, the retest interval was a mere 2-3 days. Chawalow \& Adesina [57] indicated that high test-retest correlation indices obtained over a short period ( $<1$ week) may simply be reflected memory rather than actual stability of participants' perceptions. Consequently, higher test-retest correlations do not actually reflect the stability of an instrument if the retest interval is short.

For the establishment of validity, all these instruments had one or more types of construct validity reported. Most had conducted convergent validity which met acceptable standards. Other reported approaches of validity, such as factor analysis and known-group validation, were also considered acceptable.

\section{Discussion}

There were 11 validated multidimensional QOL instruments, which could be classified into four categories: generic, cancer-specific, cancer site-specific and cancer survivor-specific instruments. All these instruments met 
the minimum requirements of reliability and validity, with the internal consistency of reliability varying from 0.68-0.99 and the test-retest reliability ranging from 0.60.95 based on the test of the Pearson coefficient. One or more types of validity supported the construct validity.

\section{General QOL measurement issues in cervical cancer survivors}

The original versions of these 11 QOL instruments were mainly developed in Europe and North America, therefore how to select those that would be most appropriate for Chinese cervical cancer survivors requires careful consideration by researchers.

Among the generic scales, the WHOQOL-BREF was the most often-used scale among QOL studies in cervical cancer survivors. Some studies chose this scale because it had been translated and validated in their language $[24,25]$. Hence, these studies chose generic scales based on practical issues. One study chose the generic scale of QLI because there was a control group from the general population [26]. Generic scales were designed and validated in the general population. If the study objectives aimed at making a comparison of QOL between cancer survivors and the general population, choosing one of the generic scales would be suitable. However, while these generic instruments may be useful for making comparisons of QOL between cervical cancer survivors and the general population, they may not be sensitive enough to detect the impact of cancer and cancer treatment on QOL among cervical cancer survivors.

The majority of QOL studies in cervical cancer survivors chose cancer-specific scales. EORTC QLQ-C30 and FACT-G were the most frequently used. It is possible that cancer-specific scales are more responsive to changes than their generic counterparts, because cancerspecific instruments cover items in addressing the effects of cancer and related treatment on QOL. In consequence, it would be logical to speculate that cancerspecific scales would be more appropriate than generic scales in assessing QOL among cancer survivors. However, this speculation is only partially substantiated. Due to a failure to identify concerns specific to cervical cancer, these instruments may not be the most suitable for assessing QOL among cervical cancer survivors.

Cancer site-specific instruments may achieve greater specificity and sensitivity than either generic or cancerspecific scales, as site-specific scales cover general cancer-specific issues and address specific concerns related to cervical cancer. There were three site-specific instruments used by studies in our review: EORTC QLQCx24, FACT-Cx and QLICP-CE. It may be speculated that these cancer site-specific scales are the most suitable choice for QOL studies in cervical cancer survivors.
Yet these scales are more concerned with the immediate effects of cancer and acute cancer treatment, so that they are not appropriate for cervical cancer survivors due to the lack of items covering the long-term sequelae of cervical cancer, such as loss of fertility, sexual dysfunction, fear of recurrence, and body image disturbance [58].

In addition, cancer survivors reported positive changes in life outlook, self-growth, precious life, and an appreciation of their relationships with others [37]. All 11 QOL instruments used in cervical cancer survivors paid less attention to the positive outcome of the cancer survivorship experience. In more recent years, the trend of QOL instrument production has continued to emerge for cancer survivors, particularly for long-term (more than 5 years) cancer survivors with an emphasis on positive outcomes [10], such as the Quality of Life Scale for Adult Cancer Survivors (QLACS) by Avis et al. [59] and the Impact of Cancer (IOC) by Zebrack et al. [60]. However, these long-term cancer survivor-specific instruments have not been applied to the population of cervical cancer survivors.

According to Ashing-Giwa's contextual model [14], socio-ecological, cultural and healthcare system-related factors are essential components in the systemic level of QOL among cancer survivors. Additional file 2 shows the paradigm, domains, components and item distribution. These 11 multidimensional QOL instruments did not adequately incorporate the contextual milieu. In other words, there were few items that captured the contextual domain of QOL, such as socio-ecological and cultural issues. Even if the instrument of WHOQOLBREF had an environmental domain, this scale covered too few items to measure the environmental domain of QOL adequately. Therefore, neither of these instruments was comprehensive enough to address or cover all QOL issues among cervical cancer survivors.

\section{Specific issues of QOL measurement in Chinese cervical cancer survivors}

Although the instruments of EORTC QLQ-C30, FACTG, and FACT-Cx had been applied to Chinese cervical cancer survivors, few studies calculated its reliability and validity when applied to Chinese women. Only one study by Wan et al. [61] established and reported FACT-G as having good reliability and validity among different types of Chinese cancer patients.

Recently, Zhang and colleagues developed the scale of QLICP-CE and validated it among a group of Chinese women with cervical cancer [36]. The domains and items included in the scale of QLICP-CE were mainly based on the instruments of EORTC QLQ-C30 and FACT-Cx. The QLICP-CE emphasized the aspects of women's appetite and sleep, as within Chinese culture 
good appetite, sleep and energy are highly regarded in daily life [62]. Due to Chinese communities viewing sex as a taboo topic, the QLICP-CE consists of just one item to measure the issues of sexual health. As sexuality is one of the essential components of QOL [63], this scale failed to address an important aspect of QOL for Chinese cervical cancer survivors.

Furthermore, culture is a major determinant of QOL, as perceptions of QOL are embedded in cultural beliefs about what constitutes normality and health [64]. At the individual level, the components of health practices, health utilization and perceived health practice should be measured in a culturally sensitive manner, because in Chinese culture the beliefs of Taoism (human beings should live in harmony with nature, that is, with 'Tao' as the way of life) and traditional Chinese medicine (TCM) (expanded from Taoism, it views health as harmony between vital energy - known as Qi - within and between the body and its environment) are dominate the views of health and health utilization $[62,65]$. These beliefs are different from those of Western people, therefore perceptions of QOL logically also differ between China and the West. Due to differences of social backgrounds and healthcare systems, at the systemic level the components of socio-economic status, access to and satisfaction with health care, and role and relationship changes should be particularly emphasized. Since the family relationship and kinship play very important roles in daily life in Chinese communities [62], roles and relationship changes due to cancer and treatment greatly influence Chinese women's QOL.

\section{Limitations of the review}

In searching for literature, the 5 electronic databases used provided a comprehensive coverage of key English and Chinese medical, nursing and health-affiliated journals. However, the titles and abstracts were screened only by the first author. In order to compensate for this limitation, all articles were screened using a checklist based on clear inclusion and exclusion criteria. Additionally, all eligible articles were agreed upon by the research team. Other limitations include that the assessment of the psychometric properties of QOL instruments was limited to the reliability and validity. This review failed to assess other instrument properties, such as cross-cultural acceptability, responsiveness, and acceptability, because those properties were seldom reported by the instrument developers.

\section{Conclusion}

According to this review, a total of 11 validated multidimensional instruments have been used to assess QOL among cervical cancer survivors. Almost all these QOL instruments were originally developed in North America or Europe. Due to cultural differences between these regions and China, further research needs to explore culturally specific issues in detail, such as what QOL domains are known to be important for Chinese women.

Regarding the issue of instrument selection, choosing an instrument for Chinese cervical cancer survivors should first take consideration of the QOL instruments' psychometric properties. Based on this review, all 11 instruments met the minimum requirements of reliability and validity. Secondly, instrument selection should be based on the purpose of investigation. From the previous discussion, if a study aims to compare the QOL of Chinese cervical cancer survivors with that of the general Chinese female population, the WHOQOL-BREF could be one of the potential instruments. By contrast, if the purpose of the study is to investigate QOL among survivors of different types of cancer including cervical cancer, QLACS, CaSUN and IOC should be translated and applied to Chinese cervical cancer survivors. Lastly, if the aim is simply to investigate QOL among short-term cervical cancer survivors, the QLICP-CE would be a potential choice.

Finally, instrument selection for Chinese cervical cancer survivors also needs to consider the comprehensiveness of the instruments. This issue could be addressed by incorporating different types of QOL instruments based on the purpose of investigation. However, choosing multiple QOL instruments, there is a high possibility that more time will be required of respondents. Consequently, further research is needed to develop an instrument tailored to assessing QOL for Chinese cervical cancer survivors across the whole survivorship, including immediately after diagnosis, in the short term (less than 5 years), and in the long term (more than 5 years).

\section{Additional file 1: Categories of the established multidimensional QOL instruments adopted by studies in cervical cancer survivors. \\ Additional file 2: The paradigm, domains, components and distribution of items across 11 multidimensional QOL instruments. \\ Additional file 3: The psychometric properties of the 11 established multidimensional QOL instruments.}

\section{Authors' contributions}

The first author was responsible for conducting the literature review and drafting the manuscript. All authors were involved in planning, reviewing, discussion, reporting, and approval of the final manuscript.

\section{Competing interests}

The authors declare that they have no competing interests.

Received: 5 October 2009 Accepted: 19 March 2010 Published: 19 March 2010

\section{References}

1. China Cancer Database: The epidemiology of cervical cancer. Beijing China [http://cancernet.cicams.ac.cn/index.php?option=content\&task=view\&id=23\& Itemid=40], Accessed on 2/11/2008. 
2. Yin $X H$, Zhou BB, Zhu CP: The impact factors and interventions on quality of life among cervical cancer patients. Med J CASC 2004, 6:78-79, [in Chinese].

3. Wolff SN: The burden of cancer survivorship: A pandemic of treatment success. Handbook of Cancer Survivorship New York: SpringerFeuerstein M 2007, 7-18.

4. Gotay C: Quality of life assessment in cancer. Handbook of Cancer Control and Behavioral Science: A Resource for Researchers, Practitioners, and Policymakers Washington, DC: American Psychological AssociationMiller SM, Bowen DJ, Croyle RT, Rowland JH 2009, 115-128.

5. Dow KH, Ferrell BR, Haberman MR, Eaton L: The meaning of quality of life in cancer survivorship. Oncol Nurs Forum 1999, 26:519-528.

6. Yang K, Yin TJC: Defining the content domain of health-related quality of life for terminally ill cancer patients. Nurs Res 1999, 7:129-144, [in Chinese].

7. Molassiotis A, Chan CWH, Yam BMC, Chan SJ: Quality of life in Chinese women with gynecological cancers. Supportive Care Cancer 2000, 8:414-422.

8. Ferrell BR, Dow KH, Leigh S, Ly J, Gulassekaram P: Quality of life in long term cancer survivors. Oncol Nurs Forum 1995, 22:915-922.

9. Vistad I, Fosså SD, Dahl AA: A critical review of patient-rated quality of life studies of long-term survivors of cervical cancer. Gynecol Oncol 2006, 102:563-572.

10. Pearce NJ, Sanson-Fisher R, Campbell HS: Measuring quality of life in cancer survivors: a methodological review of existing scales. PsychoOncology 2008, 17:629-640.

11. Zebrack BJ, Cella DF: Evaluation of quality of life in cancer survivors. Outcomes Assessment in Cancer: Measures, Methods, and Applications Cambridge: Cambridge University PressLipscomb J, Gotay CC, Snyder C 2005, 241-250.

12. Bloom JR, Petersen DM, Kang SH: Multi-dimensional quality of life among long-term (5+ years) adult cancer survivors. Psycho-Oncology 2007, 16:691-706.

13. Ferrell BR, Grant MM, Funk B, Otis-Green S, Garcia N: Quality of life in breast cancer survivors as identified by focus groups. Psycho-Oncology 1997, 6:13-23.

14. Ashing-Giwa KT: The contextual model of HRQoL: A paradigm for expanding the HRQoL framework. Qual Life Res 2005, 14:297-307.

15. American National Cancer Institute Office of Cancer Survivorship: About Cancer Survivorship Research: Survivorship Definitions. Rockville: U.S [http://cancercontrol.cancer.gov/ocs/definitions.html], (accessed on 2 November 2008).

16. Boling W, Fouladi RT, Basen-Engquist K: Health-related quality of life in gynecological oncology: Instruments and psychometric properties. Int $J$ Gynecol Cancer 2003, 13:5-14.

17. LoBiondo-Wood G, Haber J, (Eds): Nursing Research: Methods and Critical Appraisal for Evidence-Based Practice. St. Louis: Mosby, 62006.

18. DeVellis RF, (Ed): Scale Development: Theory and Application. Thousand Oaks: Sage, 22003.

19. Fitzpatrick R, Davey C, Buxton MJ, Jones DR: Evaluating patient-based outcome measures for use in clinical trials. Health Technol Assess (Winchester, England) 1998, 2:1-74

20. Steiner DL, Norman GR, (Eds): Health Measurement Scales: A Practical Guide to Their Development and Use. New York: Oxford University Press, 32003.

21. McDowell I, (Ed): Measuring Health: A Guide to Rating Scales and Questionnaires. New York: Oxford University Press 2006.

22. Brotto LA, Heiman JR, Goff B, et al: A psychoeducational intervention for sexual dysfunction in women with gynecologic cancer. Arch Sex Behav 2008, 37:317-329.

23. Capelli G, De Vincenzo Rl, Addamo A, Bartolozzi F, Braggio N, Scambia G: Which dimensions of health-related quality of life are altered in patients attending the different gynecologic oncology health care settings? Cancer 2002, 95:2500-2507.

24. Vaz AF, Pinto-Neto AM, Conde DM, Costa-Paiva L, Morais SS, Esteves SB: Quality of life of women with gynecologic cancer: Associated factors. Arch Gynecol Obstet 2007, 276:583-589.

25. Vaz AF, Pinto-Neto AM, Conde DM, Costa-Paiva L, Morais SS, Esteves SB: Quality of life and acute toxicity of radiotherapy in women with gynecologic cancer: A prospective longitudinal study. Arch Gynecol Obstet 2008, 278:215-223.
26. Rannestad T, Skjeeldestad FE, Platou TF, Hagen B: Quality of life among long-term gynecological cancer survivors. Scandinavian J Caring Sci 2008, 22:472-477.

27. Korfage IJ, Essink-Bot M, Mols F, Poll-Franse van de L, Kruitwagen R, van Ballegooijen M: Health-related quality of life in cervical cancer survivors: A population-based survey. Int I Radiat Oncol Biol Phys 2009, 73:1501-1509.

28. Frumovitz M, Sun CC, Schover LR, Munsell MF, Jhingran A, Wharton JT, et al: Quality of life and sexual functioning in cervical cancer survivors. J Clin Oncol 2005, 23:7428-7436.

29. Chan YM, Ngan HYS, Li BYG, et al: A longitudinal study on quality of life after gynaecologic cancer treatment. Gynecol Oncol 2001, 83:10-19.

30. Chen YP, Yeh SH, Kung FT, Ho MY: A correlational study of symptom distress, depression and quality of life in hospitalized cervical cancer patients. J Evi Based Nurs 2007, 3:89-98, [in Chinese].

31. Du AY, Zhang JW, Tang L, Yu MJ: Quality of life among gynecological patients after surgery. Chin Pract J Gynecol Obstet 2007, 23:198-200, [in Chinese].

32. Guo Y, Sheng XJ, Liu Y, Hua XF: Evaluation of quality of life for gynecologic cancer patients. Chin J Cancer Res 2004, 16:292-296, [in Chinese].

33. Greimel ER, Winter R, Kapp KS, Haas J: Quality of life and sexual functioning after cervical cancer treatment: a long-term follow-up study. Psycho-Oncology 2009, 18:476-482.

34. Ashing-Giwa KT: Enhancing physical well-being and overall quality of life among underserved latina-american cervical cancer survivors: Feasibility study. J Cancer Surviv 2008, 2:215-223.

35. Ashing-Giwa KT, Tejero JS, Kim J, Padilla GV, Kagawa-Singer M, Tucker MB, et al: Cervical cancer survivorship in a population based sample. Gynecol Oncol 2009, 112:358-364.

36. Zhang $X Q$, Wan $C H$, Lu YB, Yang HY, Luo JH, Huang Y, Meng $Q$ : Development and evaluation of quality of life instruments for cancer patients-Cervix Cancer (QLICP-CE). Chin Oncol 2009, 18:183-186, [in Chinese].

37. Hodgkinson K, Butow P, Fuchs A, Hunt GE, Stenlake A, Hobbs KM, Brand A, Wain G: Long-term survival from gynecologic cancer: Psychosocial outcomes, supportive care needs and positive outcomes. Gynecol Oncol 2007, 104:381-389.

38. Ware JE, Snow KK, Kosinski M, Gandek B: SF-36 Health Survey Manual and Interpretation Guide. Boston: New England Medical Center, The Health Institute 1993

39. Brazier JE, Harper R, Jones NMB, O'Cathain A, Thoma KJ, Usherwood T, Westlake I: Validating the SF 36 health survey questionnaire: a new outcome measure for primary care. BMJ 1992, 305:160-164.

40. Hsiung PC, Fang CT, Chang YY, Chen MY, Wang JD: Comparison of WHOQOL-BREF and SF-36 in patients with HIV infection. Qual Life Res 2005, 14:141-150.

41. Padilla GV, Frank-Stromborg M, Koresawa S: Single instruments for measuring quality of life. Instruments for Clinical Health-care Research Sudbury: Jones and BartlettFrank-Stromborg M, Olsen SJ , 3 2004, 128-149.

42. Grace Y, Chung CW, Yu CF, Wang JD: Development and verification of validity and reliability of the WHOQOL-BREF Taiwan version. $J$ Formos Med Assoc 2002, 101:342-351.

43. Skevington SM, Lotfy M, O'Connel KA: The World Health Organization's WHOQOL-BREF quality of life assessment: Psychometric properties and results of the international field trial-a report from the WHOQOL Group. Qual Life Res 2004, 13:299-310.

44. Ferrans C, Powers M: Psychometric assessment of the Quality of Life Index. Res Nurs Health 1992, 15:29-38.

45. Dougherty $C$, Dewhurst T, Nichol P, Spertus J: Comparison of three quality of life instruments in stable angina pectoris: Seattle Angina Questionnaire, Short Form Health Survey (SF-36), and Quality of Life Index - Cardiac Version III. J Clin Epidemiol 1998, 51:569-575.

46. Oleson M: Subjectively perceived quality of life. Image: J Nurs Scholarsh 1990, 22:187-190.

47. McDowell I, (Ed): Measuring Health: A Guide to Rating Scales and Questionnaires. New York: Oxford University Press 2006.

48. van Agt HME, Essink-Bot ML, Krabbe PFM, et al: Test-retest reliability of health state valuations collected with the Euro QoL questionnaire. Soc Sci Med 1994, 39:1537-1544. 
49. Hurst N, Jobanputra P, Hunter M, et al: Validity of EuroQol-a generic health status instrument in patients with rheumatoid arthritis. $\mathrm{Br} J$ Rheumatol 1994, 33:655-662.

50. Schag CA, Ganz PA, Heinrich RL: Cancer Rehabilitation System-Short Form (CARES-SF): A cancer specific rehabilitation and quality of life instrument. Cancer 1991, 68:1406-1413.

51. EORTC Quality of Life Group QLG): Guidelines for Developing Questionnaires Modules. Brussels: EORTC 2002 [http://groups.eortc.be/qol/ downloads/200208module_development_guidelines.pdf], Accessed on 2/ $11 / 2008$

52. Hjermstad MJ, Fossa SD, Bjordal K, et al: Test/retest study of the European Organization for Research and Treatment of Cancer Core Quality-of-Life Questionnaire. J Clin Oncol 1995, 13:1249-1254.

53. Niezgoda HE, Pater JL: A validation study of the domains of the core EORTC Quality of Life questionnaire. Qual Life Res 1993, 2:129-140.

54. Cella DF, Tulsky DS, Gray G, Sarafian B, Lloyd S, Linn E, et al: The Functional Assessment of Cancer Therapy (FACT) scale: Development and validation of the general measure. J Clin Oncol 1993, 11:570-579.

55. Greimel ER, Vlasic KK, Waldenstrom AC, et al: On behalf of the European Organisation for Research and Treatment of Cancer (EORTC) Quality of Life Group. The European Organisation for Research and Treatment of Cancer (EORTC) Quality-of-Life Questionnaire cervical cancer module, EORTC QLQ-CX24. Cancer 2006, 107:1812-1822.

56. Monk BJ, Huang HQ, Cella D, Long HJ: Quality of life outcomes from a randomized phase III trial of cisplatin with or without topotecan in advanced carcinoma of the cervix: a Gynecologic Oncology Group Study. J Clin Oncol 2005, 23:4617-4625.

57. Chawalow AJ, Adesina AB: Conception, development, and validation of instruments for quality of life assessment: An overview. Statistical Methods for Quality of Life Studies: Design, Measurement and Analysis Boston: Kluwer Academic PublishersMesbah M, Lee MLT 2002, 63-70.

58. Hewitt M, Greenfield S, Stovall E: From cancer patients to cancer survivors: Lost in transition. Washington, DC: The National Academic Press 2006.

59. Avis NE, Smith KW, McGraw S, et al: Assessing quality of life in adult cancer survivors (QLACS). Qual Life Res 2005, 14:1007-1023.

60. Zebrack B, Ganz P, Bernaards CA, Petersen L, Abraham L: Assessing the impact of cancer: development of a new instrument for long-term cancer survivors. Psycho-Oncology 2006, 15:407-421.

61. Wan $\mathrm{CH}$, Meng $\mathrm{Q}$, Tang $\mathrm{XL}$ : To evaluation of the Chinese version of FACTG among Chinese cancer patients. J Practi Oncol 2006, 21:77-80, [in Chinese].

62. Wan $\mathrm{CH}$, Yang Z, Meng Q, et al: Developing and validation of the general module of the system of quality of life instruments for cancer patients. Int J Cancer 2008, 122:190-196.

63. Krebs LU: Sexual assessment in cancer care: Concepts, methods and strategies for success. Seminars Oncol Nurs 2008, 24:80-90.

64. Padilla GV, Kagawa-Singer M: Quality of life and culture. Quality of Life: From Nursing and Patients Perspectives Sudbury: Jones and Bartlett PublishersKing CR, Hinds PS, 2 2003, 117-142.

65. Chen YC: Chinese values, health and nursing. J Adv Nurs 2001, 36:270-273.

doi:10.1186/1477-7525-8-30

Cite this article as: Zeng et al:: Quality of life measurement in women with cervical cancer: implications for Chinese cervical cancer survivors. Health and Quality of Life Outcomes 2010 8:30.

\section{Submit your next manuscript to BioMed Central and take full advantage of:}

- Convenient online submission

- Thorough peer review

- No space constraints or color figure charges

- Immediate publication on acceptance

- Inclusion in PubMed, CAS, Scopus and Google Scholar

- Research which is freely available for redistribution

Submit your manuscript at www.biomedcentral.com/submit
C Biomed Central 